\title{
Vascular endothelial growth factor in pleural effusions of different origin
}

\author{
U. Sack*, M. Hoffmann\#, X.J. Zhao*, K.S. Chan`, D.S.C. Hui ${ }^{+}$, H. Gosse ${ }^{\S}$, \\ L. Engelmann", J. Schauer", F. Emmrich* and G. Hoheisel"
}

ABSTRACT: This study aimed to determine the diagnostic relevance of vascular endothelial growth factor (VEGF) in the pleural fluid and serum of patients with pleural effusions of different aetiology.

VEGF was quantified in the pleural effusion fluid and serum of 96 patients with malignancies (58 lung cancers (CA) and 38 tumours with secondaries to the lung (TM)), 45 with congestive heart failure (CHF), 28 with tuberculosis (TB), 45 with acute infections (INF), and in the serum of 20 healthy controls.

VEGF pleural effusion concentrations were significantly different in the main diagnostic groups. VEGF was higher in effusions of patients with malignancies (CA as well as TM) in comparison with INF, TB or CHF. In serum, however, high VEGF concentrations indicated CA, TM or INF, but not TB or CHF. Despite significant differences of VEGF levels in different patient groups, receiveroperating characteristic analysis revealed insufficient diagnostic value of VEGF for differential diagnosis of pleural effusions.

In conclusion, vascular endothelial growth factor serum concentration is highly suggestive of the presence of lung disease in general, except for tuberculosis. In effusion fluid, the presence of vascular endothelial growth factor clearly indicates inflammatory or malignant origin. However, for diagnostic use, additional parameters besides vascular endothelial growth factor are mandatory.

KEYWORDS: Pleural effusion, receiver-operating characteristic analysis, serum, vascular endothelial growth factor

V ascular endothelial growth factor (VEGF), also known as vascular permeability factor, is a glycoprotein, which is able to increase the angiogenetic, growth-stimulating and permeability-enhancing properties of vascular endothelial cells [1, 2]. VEGF is expressed by a number of different tumour cell types, such as adenocarcinoma, urinary bladder carcinoma, fibrosarcoma and lymphoma cells [3]. VEGF is also expressed by the cells of normal tissues, such as the lung, kidney, adrenal gland, heart, liver and stomach, as well as in macrophages of the peritoneum [4, 5]. Differing VEGF levels were found in carcinomatous, inflammatory and tuberculous pleural effusions, implying a varying degree of influence on the process of fluid accumulation in the pleural space in different disease states [6-10]. In this study, the current authors were interested to know whether pleural effusions of different origin could be differentiated by the determination and comparison of VEGF pleural fluid concentrations. Furthermore, the current authors aimed to determine the degree of compartmentalisation by comparing VEGF concentrations in pleural effusion fluid and respective serum. Finally, receiver-operating characteristic (ROC) analysis was performed to investigate the diagnostic value of VEGF.

\section{PATIENTS AND METHODS \\ Patients}

A total of 214 patients (94 females, 120 males, mean \pm SD age $61 \pm 18$ yrs) were consecutively entered into the study. The diagnosis of malignancy in patients with lung cancer (CA) or lung secondaries with primary tumours elsewhere in the body (TM) was based on either a pleural fluid cytological examination or a pleural biopsy specimen that was positive for malignancy, or if the patient had known metastatic malignancy with no other explanation for the pleural effusion.

In the $58 \mathrm{CA}$ patients, the histological types were adenocarcinoma $(n=28)$, nonsmall cell carcinoma of undetermined type $(n=19)$, small cell carcinoma $(n=6)$ and squamous cell carcinoma $(n=5)$. The locations of the primary tumours in the 38

\section{AFFILIATIONS}

*Institute of Clinical Immunology and

Transfusion Medicine, and

\#Medical Dept, Pulmonary and

Intensive Care Unit, University of

Leipzig, and

${ }^{\S}$ Robert Koch Hospital, Leipzig,

Germany.

'Respiratory and Palliative Care Unit,

Haven of Hope Hospital, and

+Dept of Medicine and Therapeutics,

Respiratory Unit, Prince of Wales

Hospital, The Chinese University of

Hong Kong, Hong Kong, China.

CORRESPONDENCE

U. Sack

University of Leipzig

Institute of Clinical Immunology and

Transfusion Medicine

Johannisallee 30

04103 Leipzig

Germany

Fax: 493419725828

E-mail: ulrich.sack@

medizin.uni-leipzig.de

Received:

March 302004

Accepted after revision:

November 302004

SUPPORT STATEMENT

This study was supported by travel grant 423/hk-rl, Joint Research

Project Deutscher Akademischer

Austauschdienst (DAAD), Bonn,

Germany, and Research Council of

Hong Kong, Hong Kong; by

Förderverein Pneumologie, University

of Leipzig, Leipzig, Germany; and by

the Interdisziplinäres Zentrum für

Klinische Forschung (IZKF) Leipzig at

the Faculty of Medicine of the

Universität Leipzig, Leipzig, Germany

(project Z10). This study also

contains parts of the inaugural thesis by M. Hoffmann.

European Respiratory Journal

Print ISSN 0903-1936

Online ISSN 1399-3003 
TM patients with secondaries to the lung were the breast $(n=6)$, stomach $(n=2)$, rectum $(n=2)$, colon $(n=2)$, urethra $(n=2)$, kidney $(n=1)$, parotic gland $(n=1)$, leukaemia $(n=3)$, melanoma $(n=1)$, and tumours of unknown primary location $(n=18)$.

The 45 patients with effusions of infectious origin (INF) were mainly intensive care unit patients who suffered from bacterial pneumonia $(\mathrm{n}=24)$, septicaemia in part with multi-organ failure $(n=14)$, or empyema $(n=7)$.

The diagnosis of tuberculosis (TB) was based on histological evidence of granulomatous pleuritis in pleural biopsies, microbiological proof in effusion fluid or sputum, and/or on response to anti-TB therapy.

The diagnosis of the 45 patients with congestive heart failure (CHF) was based on clinical symptoms and signs, radiological evidence of cardiomegaly and congested lungs, a transudative effusion in most cases, the exclusion of other aetiologies and the response to appropriate therapy.

The serum of 20 healthy blood donors served as controls.

\section{Collection of pleural fluid and serum samples}

Following institutional guidelines, the study was approved by the ethical committees at the University of Leipzig and Robert Koch Hospital, Leipzig, Germany, and the Haven of Hope Hospital and Prince of Wales Hospital, The Chinese University, Hong Kong, China. Informed consent was obtained from each patient. Thoracocentesis was performed for diagnostic or therapeutic purposes, and pleural effusions and serum samples were collected at the time of first tapping. The pleural fluid was collected in tubes containing EDTA (final concentration of $1 \mathrm{mg} \cdot \mathrm{mL}^{-1}$ ) for determination of the cellular content and in plain tubes for determination of VEGF, lactate dehydrogenase (LDH) and total protein. The total cell, white cell and differential cell counts (May-Grünwald-Giemsa stain) were determined by counting 200 cells using light microscopy. The cell pellet and supernatant were separated by centrifugation $\left(600 \times g, 30 \mathrm{~min}, 4^{\circ} \mathrm{C}\right)$. Peripheral venous blood was collected in plain tubes for the determination of VEGF, LDH and total protein. The protein and LDH concentrations were determined in effusion fluid and in serum by standardised automated methods. Light's criteria were calculated to differentiate exudative from transudative effusions [11].
Serum and effusion fluids were stored at $-70^{\circ} \mathrm{C}$ for later VEGF analysis.

\section{Laboratory analysis}

ELISA for human VEGF was performed on all serum and pleural effusion samples, using the commercially available Quantikine $^{\mathrm{TM}}$ kit (R\&D Systems GmbH, Wiesbaden, Germany). ELISA plates were coated with specific mouse monoclonal antibodies directed against VEGF. The lower limit of detection, as stated by the supplier, was $5.0 \mathrm{pg} \cdot \mathrm{mL}^{-1}$. After standards and cell-free samples were pipetted into the wells of the microtitre plates, specific horseradish peroxidase-linked polyclonal antibodies were added and immunoreactive levels of VEGF determined. Values below the detection limit were assumed as zero for statistical analyses.

\section{Statistical analysis}

Data are presented means \pm SD. Data were checked on homogeneity (Levene test). Significant differences were identified by ANOVA, followed by Tuckey or Tamhane tests for homogeneously or nonhomogeneously distributed data sets, respectively. Correlations were determined by Pearson's test. All p-values $<0.001$ were considered statistically significant. ROC curves and the related areas under the curve were calculated using online-provided data analysis tools from Acomed (Acomed, Leipzig, Germany).

\section{RESULTS \\ Characteristics of study groups}

Table 1 depicts the different aetiologies and characteristics of the study patients. No statistical differences between the diagnostic groups were detected for age and sex, even in the comparatively younger control group. The percentage of smokers in the CA patients was higher in comparison with other groups as expected. No difference for all parameters, however, was observed when the smokers and nonsmokers from each study group were compared.

\section{General characteristics of samples collected}

Using Light's criteria, transudates and exudates can be differentiated according to their ratios of serum and effusion protein concentrations and respective LDH concentrations [11]. The respective mean ratios of the study groups met these criteria (table 2$)$. However, only 35 out of 45 patients $(78 \%)$

TABLE 1 Characteristics of study groups

\begin{tabular}{lcccc} 
Diagnosis & Subjects $\mathbf{n}$ & Males/females & Age yrs & Smoker/nonsmoker \\
\hline Healthy controls & 20 & $12 / 8$ & $35.1 \pm 9.6$ & $11 / 9$ \\
Lung cancer & 58 & $38 / 20$ & $68.9 \pm 11.4$ & $40 / 18$ \\
TM & 38 & $11 / 27$ & $67.6 \pm 14.9$ & $6 / 32$ \\
Empyema/pneumonia/septicaemia & 45 & $37 / 8$ & $57.7 \pm 17.2$ & $11 / 34$ \\
Tuberculosis & 28 & $23 / 5$ & $50.9 \pm 21.1$ & $12 / 16$ \\
Congestive heart failure & 45 & $19 / 26$ & $70.7 \pm 9.5$ & $22 / 23$ \\
Total & 234 & $140 / 94$ & $61.0 \pm 18.0$ & $102 / 132$ \\
\hline
\end{tabular}

Data are presented as $n$ and mean $\pm S D$. TM: tumours with secondaries to the lung. 


\begin{tabular}{|c|c|c|c|c|c|c|}
\hline & Controls & CA & TM & INF & TB & CHF \\
\hline Percentage of exudates $\%$ & & 95 & 92 & 80 & 93 & 22 \\
\hline $\begin{array}{l}\text { Leukocytes in effusion } \cdot \mu \mathrm{L}^{-1} \\
\text { Significant differences }\end{array}$ & & $\begin{array}{c}6412 \pm 13702 \\
\#\end{array}$ & $1963 \pm 2390$ & $5873 \pm 8975$ & $\stackrel{3221 \pm 3535}{\#}$ & $\underset{-330 \pm 227}{-f}$ \\
\hline $\begin{array}{l}\text { Protein ratio effusion/serum } \\
\text { Significant differences }\end{array}$ & & $\begin{array}{c}0.657 \pm 0.212 \\
\#\end{array}$ & $\begin{array}{c}0.690 \pm 0.213 \\
\#\end{array}$ & $\begin{array}{c}0.497 \pm 0.165 \\
\#\end{array}$ & $\begin{array}{c}0.723 \pm 0.136 \\
\#\end{array}$ & $\begin{array}{l}0.318 \pm 0.143 \\
\uparrow,+, s, f\end{array}$ \\
\hline $\begin{array}{l}\text { VEGF } \mathbf{p g} \cdot \mathbf{m L}^{-1} \text { in serum } \\
\text { Significant differences }\end{array}$ & $\begin{array}{l}51 \pm 21 \\
\bullet,+, \$\end{array}$ & $\begin{array}{c}500 \pm 410 \\
\# \#\end{array}$ & $\begin{array}{c}405 \pm 291 \\
\# \#\end{array}$ & $\begin{array}{c}660 \pm 536 \\
\# \#, \#\end{array}$ & $362 \pm 268$ & $\begin{array}{c}290 \pm 248 \\
\#\end{array}$ \\
\hline $\begin{array}{l}\text { VEGF pg } \cdot \mathbf{m L}^{-1} \text { in effusion } \\
\text { Significant differences }\end{array}$ & & $\begin{array}{c}2349 \pm 2910 \\
s, f . \#\end{array}$ & $\begin{array}{c}2183 \pm 2648 \\
s, f, \#\end{array}$ & $\begin{array}{l}955 \pm 1488 \\
f,+, f, \#\end{array}$ & $\underset{\uparrow+,+5}{762 \pm 936}$ & $\underset{\uparrow}{196 \pm 532}$ \\
\hline
\end{tabular}

with CHF effusions met the criteria for a transudate, probably due to the different time period between the appearance of effusion until diagnosis and tapping, or due to preceding diuretic therapy ("pseudo-exudate"). Likewise, patients, although comparatively smaller in number, expected to have exudates in cases of malignancy or infection in fact exhibited criteria for a transudate, most likely due to a component of accompanying heart failure or different time periods between the onset of effusion, diagnosis and tapping (table 2).

The classification of patients in this study was, therefore, primarily based on the clinical diagnosis rather than on Light's criteria alone. Following the current authors' observations, it is believed that the VEGF value in such a patient would be closer to clinical reality and, therefore, patients were not excluded from a diagnostic group when the expected criteria by Light were not met.

\section{VEGF in sera and effusions}

No dependency of VEGF concentrations from age, sex or smoking history of the patient and control groups investigated could be observed.

In serum, healthy blood donors exhibited only very low VEGF concentrations and were therefore different from patient groups with CA, TM or INF, but not from those with TB or $\mathrm{CHF}$ (table 2). Furthermore, INF caused significantly higher VEGF concentrations in serum in comparison with $\mathrm{CHF}$ patients. All other groups were not different from one another.

The highest VEGF concentrations in pleural effusions were found in malignancies allowing differentiation from all other entities, but with no significant differences between CA and TM. Except for TB and CHF effusions, all diagnostic groups could be differentiated from one another with adequate accuracy (table 2).
Adjustment of VEGF concentrations to the protein content of effusions negatively influenced significancy levels between tumours (CA as well as TM) and CHF. This was also true for other comparisons. Therefore, the respective ratios were not calculated further (data not shown).

The calculation of ratios between VEGF levels in effusions and sera confirmed differences between malignancies (CA and TM) in comparison with all other patient groups (TB, INF and CHF; table 2).

Of all the investigated cellular parameters, only leukocytes were significantly different between $C A$ and $T B$, in comparison with $\mathrm{CHF}$ but not total cells, lymphocytes, monocytes, or granulocytes (data not shown). Interestingly, the leukocyte counts in effusions correlated with VEGF concentrations.

\section{Receiver-operating characteristic analysis of VEGF}

To investigate the potential of VEGF determination for diagnostic use, ROC curves were calculated for the differential diagnosis of malignancies (CA and TM) causing pleural effusions (fig. 1). Although the specificity could be enhanced up to $95 \%$ (at $29 \%$ sensitivity level), the best sensitivity of an acceptable $80 \%$ level had a specificity of only $58 \%$.

\section{DISCUSSION}

This study confirms that VEGF is found in pleural effusions of different origin. The highest VEGF levels were found in effusions of patients suffering from CA and TM elsewhere in the body. In addition, lower VEGF concentrations in TB effusions could be confirmed. Effusions rich in leukocytes correlated with higher VEGF levels. Mean VEGF levels in exudates were significantly higher than corresponding mean VEGF levels in serum. 


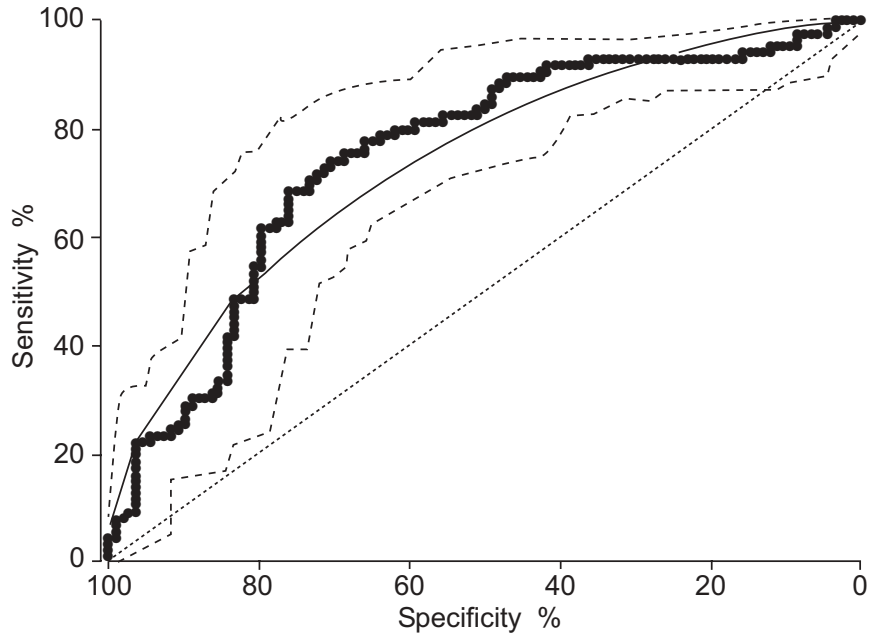

FIGURE 1. Receiver-operating characteristics (ROC) curve (๑) for differential diagnosis of tumours (lung cancer and tumours with secondaries to the lung) by vascular endothelial growth factor in pleural effusions. The fitted ROC curve is represented by (_- ). The graph also shows the 90\% confidence interval ( - - - ) and the hypothetical ROC curve resulting from homogenous distributed data (.....). These data indicate 22,26 or $55 \%$ sensitivity at 95,90 or $80 \%$ specificity, respectively.

In the pleural compartment not only do mesothelial cells, infiltrating inflammatory cells, and, in malignant pleuritis, cancer cells, but also adjacent airway epithelial cells, type II alveolar cells, alveolar macrophages, infiltrating neutrophils and eosinophils of the lung contribute to the VEGF accumulation in pleural fluid [12-16]. VEGF has potent angiogenic, mitogenic and vascular permeability-enhancing properties that are specific for endothelial cells [17]. As an important regulator of tumour angiogenesis in vivo, VEGF exerts its effects on the endothelial cell membrane [18]. Recent studies have demonstrated high levels of VEGF in exudative inflammatory and neoplastic pleural effusions, and a possible aetiological role in the pathogenesis of these types of effusions $[6,17,19]$. Pleural fluid VEGF is biologically active and may promote tumour growth and chemotaxis [13]. VEGF levels in pleural effusions are significantly correlated with the levels of tumour growth factor- $\beta$ [19], which is one of the factors considered to regulate VEGF accumulation in the pleural space [12]. In acute respiratory distress syndrome, VEGF seems to be downregulated as a protective mechanism aimed at limiting endothelial permeability [16]. Other results suggest that bacterial pathogens induce VEGF release in mesothelial cells and alter mesothelial permeability, leading to protein exudation in empyema [20]. Furthermore, VEGF mRNA has been described as being upregulated by increased ventilation caused by hypoxia or hypercapnia [21].

The observation from the current study that effusions rich in leukocytes were correlated with higher VEGF levels is known from other studies where high cellularity haemorrhagic effusions showed significantly higher VEGF levels compared with other effusion types [22]. Similar findings are known from pro-inflammatory cytokines, which correlate with VEGF, especially in TB pleurisy $[10,23]$. The current finding that the mean VEGF level in exudates was significantly higher than mean VEGF level in serum indicates local VEGF accumulation within the pleural cavity, as described in a previous study [24].

Furthermore, the current data indicate significant differences between vascular endothelial growth factor concentrations in patients suffering from malignancies and other lung diseases. To evaluate the clinical relevance of this parameter, receiveroperating characteristic curves were calculated. In contrast to descriptive and comparing statistical methods, the analysis by receiver-operating characteristic curves allows the estimation and verification of the diagnostic suitability of a chosen parameter. The receiver-operating characteristic curves, therefore, provided an alternative to sensitivity and specificity testing, allowing the examination of a parameter's ability to discriminate between two populations, regardless of the cut-off level selected [25]. Therefore, this analysis was included to assess the overall diagnostic value of vascular endothelial growth factor concentrations in clinical practice. Although significant differences could be shown, receiver-operating characteristic analysis did not reveal vascular endothelial growth factor to have enough diagnostic power when used as a singular parameter. However, in combination with other parameters, such as cytokines or proteinases, known to reflect the origin of pleural effusions [26-29], the differential diagnosis of effusions might be further improved by including vascular endothelial growth factor concentrations into the diagnostic armentarium available to the clinician.

\section{REFERENCES}

1 Senger DR, Galli SJ, Dvorak AM, Perruzzi CA, Harvey VS, Dvorak HF. Tumor cells secrete a vascular permeability factor that promotes accumulation of ascites fluid. Science 1983; 219: 983-985.

2 Ferrara N, Henzel WJ. Pituitary follicular cells secrete a novel heparin-binding growth factor specific for vascular endothelial cells. Biochem Biophys Res Commun 1989; 161: 851-858.

3 Senger DR, Van de WL, Brown LF, et al. Vascular permeability factor (VPF, VEGF) in tumor biology. Cancer Metastasis Rev 1993; 12: 303-324.

4 Berse B, Brown LF, Van de WL, Dvorak HF, Senger DR. Vascular permeability factor (vascular endothelial growth factor) gene is expressed differentially in normal tissues, macrophages, and tumors. Mol Biol Cell 1992; 3: 211-220.

5 Monacci WT, Merrill MJ, Oldfield EH. Expression of vascular permeability factor/vascular endothelial growth factor in normal rat tissues. Am J Physiol 1993; 264: C995-C1002.

6 Yanagawa H, Takeuchi E, Suzuki Y, Ohmoto Y, Bando H, Sone S. Vascular endothelial growth factor in malignant pleural effusion associated with lung cancer. Cancer Immunol Immunother 1999; 48: 396-400.

7 Yeo KT, Wang HH, Nagy JA, et al. Vascular permeability factor (vascular endothelial growth factor) in guinea pig and human tumor and inflammatory effusions. Cancer Res 1993; 53: 2912-2918.

8 Cheng D, Rodriguez RM, Perkett EA, et al. Vascular endothelial growth factor in pleural fluid. Chest 1999; 116: 760-765. 
9 Thickett DR, Armstrong L, Millar AB. Vascular endothelial growth factor (VEGF) in inflammatory and malignant pleural effusions. Thorax 1999; 54: 707-710.

10 Hamed EA, El Noweihi AM, Mohamed AZ, Mahmoud A. Vasoactive mediators (VEGF and TNF-alpha) in patients with malignant and tuberculous pleural effusions. Respirology 2004; 9: 81-86.

11 Light RW, Macgregor MI, Luchsinger PC, Ball WC Jr. Pleural effusions: the diagnostic separation of transudates and exudates. Ann Intern Med 1972; 77: 507-513.

12 Gary L, Melkerneker D, Thompson PJ, Light RW, Lane KB. Transforming growth factor beta induces vascular endothelial growth factor elaboration from pleural mesothelial cells in vivo and in vitro. Am J Respir Crit Care Med 2002; 165: 88-94.

13 Grove CS, Lee YC. Vascular endothelial growth factor: the key mediator in pleural effusion formation. Curr Opin Pulm Med 2002; 8: 294-301.

14 Nishigaki Y, Fujiuchi S, Yamazaki Y, et al. Increased vascular endothelial growth factor in acute eosinophilic pneumonia. Eur Respir J 2003; 21: 774-778.

15 Koyama S, Sato E, Tsukadaira A, et al. Vascular endothelial growth factor mRNA and protein expression in airway epithelial cell lines in vitro. Eur Respir J 2002; 20: 1449-1456.

16 Maitre B, Boussat S, Jean D, et al. Vascular endothelial growth factor synthesis in the acute phase of experimental and clinical lung injury. Eur Respir J 2001; 18: 100-106.

17 Clifford CA, Hughes D, Beal MW, et al. Plasma vascular endothelial growth factor concentrations in healthy dogs and dogs with hemangiosarcoma. J Vet Intern Med 2001; 15: 131-135.

18 Aoki Y, Tosato G. Vascular endothelial growth factor/ vascular permeability factor in the pathogenesis of primary effusion lymphomas. Leuk Lymphoma 2001; 41: 229-237.

19 Cheng D, Lee YC, Rogers JT, et al. Vascular endothelial growth factor level correlates with transforming growth factor-beta isoform levels in pleural effusions. Chest 2000; 118: 1747-1753.

20 Mohammed KA, Nasreen N, Hardwick J, Logie CS, Patterson CE, Antony VB. Bacterial induction of pleural mesothelial monolayer barrier dysfunction. Am J Physiol Lung Cell Mol Physiol 2001; 281: L119-L125.

21 Siafakas NM, Jordan M, Wagner H, Breen EC, Benoit $\mathrm{H}$, Wagner PD. Diaphragmatic angiogenic growth factor mRNA responses to increased ventilation caused by hypoxia and hypercapnia. Eur Respir J 2001; 17: 681-687.

22 Ishimoto O, Saijo Y, Narumi K, et al. High level of vascular endothelial growth factor in hemorrhagic pleural effusion of cancer. Oncology 2002; 63: 70-75.

23 Momi H, Matsuyama W, Inoue $\mathrm{K}$, et al. Vascular endothelial growth factor and proinflammatory cytokines in pleural effusions. Respir Med 2002; 96: 817-822.

24 Ziora D, Sielska S, Dworniczak S, Oklek K, Kozielski J. [VEGF (vascular endothelial growth factor) concentration in serum and pleural fluid of patients with pleural malignancy and pleural tuberculosis]. Pneumonol Alergol Pol 2002; 70: 458-467.

25 Beck JR, Shultz EK. The use of relative operating characteristic (ROC) curves in test performance evaluation. Arch Pathol Lab Med 1986; 110: 13-20.

26 Chomej P, Bauer K, Bitterlich N, et al. Differential diagnosis of pleural effusions by fuzzy-logic-based analysis of cytokines. Respir Med 2004; 98: 308-317.

27 Hoheisel G, Sack U, Hui DS, et al. Occurrence of matrix metalloproteinases and tissue inhibitors of metalloproteinases in tuberculous pleuritis. Tuberculosis (Edinb) 2001; 81: 203-209.

28 Hoheisel G, Izbicki G, Roth M, et al. Proinflammatory cytokine levels in patients with lung cancer and carcinomatous pleurisy. Respiration 1998; 65: 183-186.

29 Hoheisel G, Zheng L, Teschler H, Striz I, Costabel U. Increased soluble CD14 levels in BAL fluid in pulmonary tuberculosis. Chest 1995; 108: 1614-1616. 